Research in Astron. Astrophys. Vol. No. , 000-000

http://www.raa-journal.org http://www.iop.org/journals/raa

Research in

Astronomy and

Astrophysics

Received [year] [month] [day]; accepted [year] [month] [day]

......

Key words: stars: supernova — stars: neutron

\title{
The supernova remnant CTB 37B and its associated magnetar CXOU J171405.7-381031: evidence for a magnetar-driven remnant
}

\author{
J. E. Horvath ${ }^{1}$ and M. P. Allen ${ }^{2}$ \\ 1 Instituto de Astronomia, Geofísica e Ciências Atmosféricas - Universidade de São Paulo, Rua do \\ Matão, 1226, 05508-900, Cidade Universitária, São Paulo SP, Brazil; foton@astro.iag.usp.br \\ 2 CEFET São Paulo, Rua Pedro Vicente 625, 01109-010, Canindé, São Paulo SP, Brazil
}

\begin{abstract}
We discuss in this Letter the association of the candidate magnetar CXOU J171405.7-381031 with the supernova remnant CTB 37B. The recent detection of the period derivative of the object allowed an estimation of a young characteristic age of only $\sim 1000 \mathrm{yr}$. This value is too small to be compatible even with the minimum radius of the remnant $\geq 10 p c$, the value corresponding to the lower limit of the estimated distance of $10.2 \pm 3.5 \mathrm{kpc}$, unless the true distance happens to be even smaller than the lower limit. We argue that a consistent scenario for the remnant origin, in which the latter is powered by the energy injected by a young magnetar, is indeed more accurate to explain the young age, and points out to its non-standard (i.e. magnetar-driven) nature.
\end{abstract}

\section{INTRODUCTION}

Firmly placed inside the population of young neutron stars, the sub-class of high-B field sources, currently composed of the Anomalous X-ray Pulsars and the Soft-Gamma Repeaters has been well identified and intensively studied over the last decades (Woods \& Thompson 2006). However, there are many puzzles concerning their progenitors and birth events. Given their short characteristic ages $\left(\tau=\frac{P}{2 \dot{P}}\right)$, they should still be associated with SN remnants. Based on positional coincidence, similarity of ages and other observable features only a few associations remain undisputed (Gaensler et al. 2001, Ankay et al. 2001, Mardsen et al. 2001, Allen \& Horvath 2004). This topic may hide clues for a proper understanding of their nature and evolution, towards an explanation of the apparent high value of the magnetar magnetic fields.

While the evolution of conventional SNR has been widely discussed in the literature (see, for instance, Truelove \& McKee 1999), it has been argued that the very formation of a highly magnetized object on short timescales should lead to a dynamical behavior of the remnant, hereby termed magnetardriven SNR. This modified dynamics, in turn, has implications for the proposed associations and should be taken into account for a consistent picture. We shall review the fundamental ideas in section 2 and, for the sake of definiteness, and apply them to the recently proposed candidate CXOU J171405.7-381031 in CTB 37B, identified as a magnetar by two different groups (Sato et al. 2010, Halpern \& Gotthelf $2010 \mathrm{~b})$. We will argue that a short derived characteristic age $\sim 1000 \mathrm{yr}$ for the magnetar poses problems for a "standard" (i.e. not driven by energy injection of the magnetar), and actually supports the magnetar-driven picture. 


\section{ENERGY INJECTION IN MAGNETAR-DRIVEN SNR}

In spite of several decades of work, the problem of gravitational collapse explosions is not solved (Burrows \& Nordhaus 2009). A sequence of events along the collapse has been well established, but substantial difficulties remain concerning the detailed mechanism(s) of the explosion, and the role (if any) of the formed compact object. Several studies of the expansion of remnants in different ISM were performed over the years, just assuming that the explosion is successful, and an energy of $\sim 10^{51} \mathrm{erg}$ (Hamuy 2003) is released in a point-like region. Of course if the central object is to become decoupled for the outgoing gas, this approach makes sense. However, such vision was first challenged by Ostriker and Gunn (1971) when they postulated that the rotation energy of a central pulsar may drive the supernovae. Later it became clear that this phenomenon is too slow to power explosions, but many features of it still remain in the so-called magnetodynamical mechanisms (Moiseenko, Bisnovatyi-Kogan \& Ardeljan 2010). A recent work addressing the lightcurve and energetics issue for the specific case of magnetars can be found in Woosley (2010).

The discovery of superstrong magnetic fields posed yet another related problem to the explosion scenario. If the field had to be amplified from an initial seed, then the pre-supernova progenitors had to possess a suitable distribution of both magnetic field and angular momentum. If the initial rotation is not fast enough, the amplification is quenched (Thompson, Chang, \& Quataert 2004). This leads to an idea that fast rotating magnetars should be born to grow their fields, although their braking is efficient in the aftermath following their birth. Thus, if a magnetar is in turn formed inside the remnant immediately after the explosion by dynamo amplification, the injection of energy (much in the same way as Ostriker and Gunn 1971 envisioned) is inevitable, and an initial energy loss $L_{0}=3.85 \times$ $10^{47}\left(\frac{B}{10^{14} G}\right)^{2}\left(\frac{1 m s}{P_{0}}\right)^{4} \operatorname{ergs}^{-1}$ and initial timescale for deceleration $\tau_{0}=0.6\left(\frac{10^{14} G}{B}\right)^{2}\left(\frac{P_{0}}{1 m s}\right)^{2} d$ can be defined for this process (Allen \& Horvath 2004). These estimates are strictly valid for constant values of the magnetic field, whereas it is clear that the very phenomenon of the field growth is operating here. However, in the absence of a full detailed treatment, the expressions above may only be used to indicate the right order, but not high accurateness, of the expected injection.

Since the injected energy scales as $B^{-2}$ and the initial $m s$ periods are required for field amplification to operate, it follows that the timescale for a substantial energy injection (that is, an energy comparable to the kinetic energy of the explosion itself, adopted to be $10^{51} \mathrm{erg}$ ) is in fact very short, of the order of $\sim$ hours (a "normal" pulsar would do so in $\sim 100-1000 \mathrm{yr}$ ). Therefore we may consider the injection as quite instantaneous on astronomical explosion standards. In short, the very formation of the magnetar leads to expect that a large fraction of the kinetic energy of the remnant would be in fact provided by the injection (see also Woosley 2010), and thus to a modified dynamical behavior at later times.

This injected energy will make a very young remnant to expand more than the corresponding case without energy injection, making it look older in the free-expansion phase (lasting for just $\sim 100 \mathrm{yr}$. The reason for that feature is the form of the injection term, later the same energy would affect the Sedov-Taylor phase, when the internal energy of the gas inside the cavity $U$ picks up a term, becoming

$$
U=E-\frac{9}{32} M \dot{R}^{2}-\frac{L_{0}}{t^{-1}+\tau_{0}^{-1}}
$$

where $R$ the radius of the SNR and $M$ the mass in motion, and the last term represents the injected energy due to the magnetar formation.

The results of these considerations were shown and discussed in Allen \& Horvath (2004). A variety of works dealing with standard SNR dynamics were then used to compare the results and point out the differences (ex. Truelove \& McKee 1999, Luz \& Berry 1999, van der Swaluw et al. 2001). Besides the mentioned quicker expansion in the free-expansion phase, the Sedov-Taylor is also modified to last longer than the case without injection, and ending after $\sim 2 \times 10^{4} E_{51}^{3 / 14} n^{-4 / 7} \mathrm{yr}$ as a leading term (Allen \& Horvath 2004), when the SNR enters a "snowplow" (radiative) phase, not very relevant to our 
problem because of the young ages expected. After recalling these main results, we believe there are good reasons to keep in mind them when SGR-AXP are tentatively associated with SNRs. A specific search for these effects was undertaken by Vink \& Kuiper (2006), with negative results. In our view, this is not surprising: after $\sim 1000 \mathrm{yr}$ the speed of the ejecta is essentially the same for models with or without energy injection (Fig. 2 of Allen \& Horvath 2004), making a kinematical identification more difficult. Therefore it is only for very young remnants that a sensible difference could be found. There are reports in the literature (see Nomoto et al. 2010 and references therein) of highly energetic supernovae (termed "hypernovae") featuring $E_{e j} \geq 10^{52} \mathrm{erg}$, but these have been identified with the progenitors of GRBs, not necessarily the same events as the birth of magnetars (however, see Yu, Cheng \& Cao 2010 for a unifying model). Even in the magnetar-birth events, there are reasons to believe that the total energy output is not large (Dall'Osso, Shore \& Stella 2009), and therefore the failure to identify hypernovae around SGR-AXP is, in principle, quite justified.

\section{THE CTB 37B-CXOU J171405.7-381031 ASSOCIATION}

The suggestion of the association of CXOU J171405.7-381031 with the remnant CTB 37B was made some time ago in Halpern \& Gotthelf (2010a), and offers a new opportunity to understand the birth of magnetars and their supernovae. These association has now been confirmed by the measurements of the $\dot{P}$ that has been measured by Sato et al. (2010) and Halpern \& Gotthelf (2010b), qualifying the central object as a magnetar. Moreover, the characteristic age is $\sim 1000 \mathrm{yr}$ within a very small range, as found by the two groups. A full discussion of the CTB 37B SNRs has been addressed in several works (Aahronian et al. 2008a, 2008b, Halpern \& Gotthelf 2010b). As in many other cases, the distance to the remnant is uncertain $(10.2 \pm 3.5 \mathrm{kpc}$; Caswell et al. 1975). It seems safe to assume, taking the angular diameter and the smallest value of the distance range, that $R>10 p c$, and possibly a figure as big as $20 p c$ for the largest distance scale.

Conventional models of SNR expansion (Truelove \& McKee 1999) run into trouble to explain such radii for an age of only 1000yr. Typically, a thousand-year old remnant will not enter the Sedov-Taylor phase before $\sim 1400 \mathrm{yr}$ unless the ejected energy is larger than expected, but in any case this would have a small effect on the radius since the latter scales as $R_{S T}=$ $15 p c\left(E_{e j} / 10^{5} 1 \mathrm{erg}\right)^{1 / 5}\left(n_{I S M} / 1 \mathrm{~cm}^{-3}\right)^{-1 / 5}\left(t / 10^{4} \mathrm{yr}\right)^{2 / 3}$, with $n_{I S M}$ the particle density of the interstellar medium in which the SNR expands and $t$ its age. It follows that the remnant can be larger than $\sim 10 p c$, but only well after 1000yr. This is at odds with the derived age of CXOU J171405.7-381031 unless the actual radius happens to be $\sim 5 p c$ because a factor of $\geq 2$ error in the distance, which seems unlikely. Fig. 1 displays the Radius-Age expected from SNRs for the two cases, a "standard" expansion and a "magnetar-driven" expansion, for the same energy $10^{51} \mathrm{erg}$ and assuming a value of $n_{I S M}=1 \mathrm{~cm}^{-3}$ for the particle density of the ISM.

As it can be checked from Fig. 1, the new data (Sato et al. 2010, Halpern \& Gotthelf 2010b) leads to a young age for the "magnetar-driven" case only (upper dotted curve), and in fact also favors the shorter of the estimated distances. Insistence on the "standard" expansion scenario would need a much shorter distance to the source, as stated above, and also the remnant to be in the free-expansion phase. Since there is information available about the density in the work of Aharonian et al. (2008b), $n_{I S M} \sim$ $0.5 \mathrm{~cm}^{-3}$ introducing a negligible shift in the curve (in fact, indistinguishable in a log plot), we believe it is fair to consider the upper curve of Fig. 1 as accurate and conclude that a magnetar-driven scenario for the expansion is implied. In other words, a low density of the ISM can not be invoked to explain the large radius of the remnant within a "standard" explosion, hence the solution of the quandary points towards the dynamics univocally.

\section{DISCUSSION AND CONCLUSIONS}

We have discussed the association of the magnetar candidate CXOU J171405.7-381031 with the CTB 37B remnant, with the aim of contributing to the identification of progenitor of these exotic objects, since the latter are still largely unknown. An initial expectation of high-mass progenitors $\left(>30-40 M_{\odot}\right)$ 


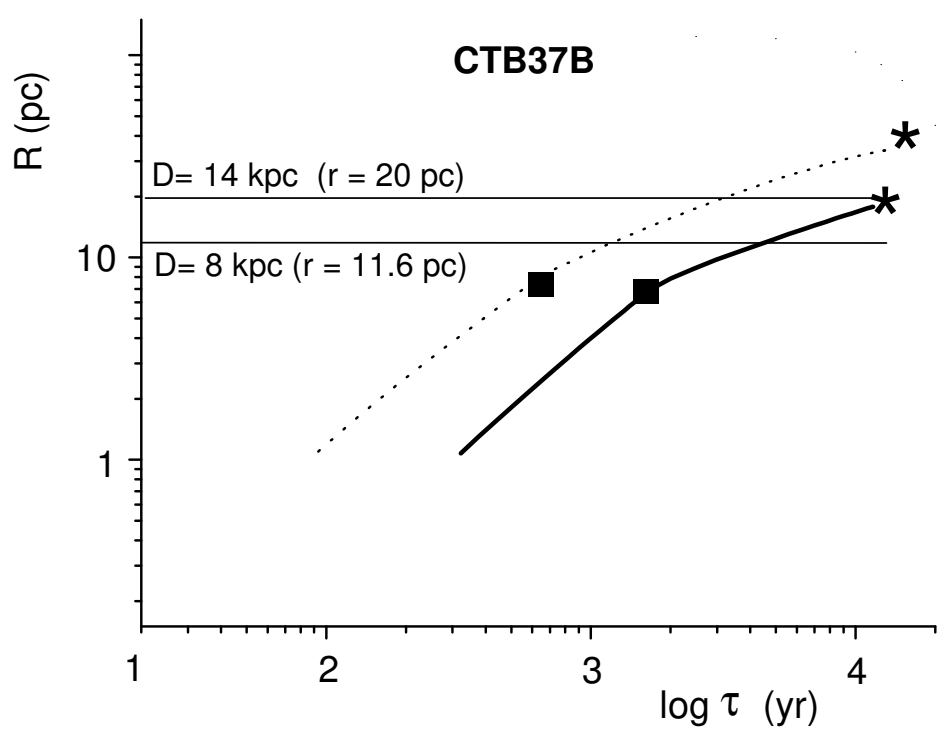

Fig. 1 Expansion of "standard" (lower solid curve) and "magnetar-driven" (upper dotted curve) remnants. The two curves correspond to identical $E_{e j}=10^{51} \mathrm{erg}$ and external densities $n_{I S M}=1 \mathrm{~cm}^{-3}$. As explained in the text and in Allen \& Horvath (2004), the difference in the solutions leads to entering before in the Sedov-Taylor phase in the latter case, and therefore the remnants expand faster than in the standard case. The transition of the free-expansion to the Sedov-Taylor phase is marked with a black square on each curve. The end of the SedovTaylor phase happens at the points marked with an asterisk. This is important to match the CTB 37B age with the derived $\tau=1000 \mathrm{yr}$ of the associated magnetar CXOU J171405.7381031, which acts as a relative chronometer. It can be checked that the matching is quite difficult for the "standard" expansion, or if the distance is closer to the extreme upper limit derived by Caswell et al. (1975) (horizontal lines, labelled).

Gaensler et al. (2005), later supported by the cluster analysis by Figer et al. (2005), Muno et al. (2005) and Bibby et al. 2008; has recently been challenged by the identification of SGR 1900+14 by Davies et al. (2009) with the $\mathrm{Cl} 1900+14$ cluster, implying a progenitor of $\leq 17 M_{\odot}$ for the cluster magnetar. If the interpretation of these observations holds, the difference between the events producing pulsars and magnetars should not be related to the mass of the progenitor, but to some other physical/evolutionary feature(s).

Our view of the problem is that, given the difficulties for growing magnetic fields to the $10^{14}-10^{15} \mathrm{G}$ scale, the dynamo scenarios should operate. They lead in turn to predict a large energy injection from the central object into the remnant, and therefore a dynamical behavior of the SNR which has to be considered with care. In other words, due to the injection of energy by the central object (a combined effect of the high rotation and the growing field, as required by $\alpha-\Omega$ dynamos) we do not deal with ordinary remnants, but rather with a very special variety of them, the magnetar-driven ones. It is in this framework that the association needs to be analyzed, as reflected not only by the finding of an energetic magnetar inside, but also by the difficulties of a standard SNR expansion of only $\sim 1000 \mathrm{yr}$ to match the observed features. A self-consistency arises from the presence of a magnetar born at the explosion: its presence reinforces a modified dynamics employment and explains its size at a young age. Note that this 
is achieved with the same total energy as a "standard" event, namely $10^{51} \mathrm{erg}$. If a larger scale is ever detected around a magnetar, its expansion would dramatically show effects of the modified dynamics, and would allow a refined test of this scenario.

Regarding the energy injection calculated to power the modified dynamics, it could be much smaller if, for example, the rapidly spinning object could get rid of its energy by gravitational radiation or other form of "invisible" (i.e not coupled to the remnant) emission. In spite that quadrupole GW would not compete with dipole losses unless the oblateness happens to be extreme (Allen \& Horvath 2004), and that it now agreed that $r$-mode excitation is not important in these situations (Watts \& Andersson 2002 , Rezzolla et al. 2001), this possibility can not be completely discarded. However, if this possibility is realized, we would learn that the birth of magnetars should be strong GW burst sources and the problem of how to amplify the magnetic field would be back.

Using the same reasoning, we have inferred before (Allen \& Horvath 2011) a range of ages for CTB 37B much smaller than the values derived within conventional models. Refining now the broad interval to include a ISM density closer to usual values (as directly measured for CTB 37B by Aharonian et al. 2008b), the numbers for the ages are still low and would not change much unless the mass of the progenitor was 3-4 times the $10 M_{\odot}$ value, but at the price of increasing the disagreement with the characteristic age of CXOU J171405.7-381031. Note the age also favors a "light" $\left(\sim 10 M_{\odot}\right)$ progenitor. Moreover, the new age is closer to the old estimation of $\sim 1500 \mathrm{yr}$ by Clark \& Stephenson (1975), but for quite different reasons. This new age allowed a prediction of the $\dot{P}$ value, $\dot{P} \sim 4 \times 10^{-11} \mathrm{~s} \mathrm{~s}^{-1}$, which in turn predicted a magnetic field strength of $B=4 \times 10^{14} G$, before the actual discovery to CXOU J171405.7-381031. It is also interesting to note that younger objects ease the requirements for energizing the TeV scale, as observed by the HESS Collaboration (Aharonian et al. 2008b): electrons have to "live" less without being cooled (Halpern \& Gotthelf 2010a) or even that the SNRs themselves contribute to the emission, because they are actually younger than they seem when their ages are erroneously estimated from conventional SNR expansion models.

Acknowledgements We acknowledge the financial support received from the Fundação de Amparo à Pesquisa do Estado de São Paulo. J.E.H. wishes to acknowledge the CNPq Agency (Brazil) for partial financial support.

\section{References}

Aharonian, F., et al. (H.E.S.S. Collaboration) 2008a, A\&A, 486, 829

Aharonian, F., et al. (H.E.S.S. Collaboration) 2008b, A\&A, 477, 353

Ankay, A., Guseinov, O. H., Alpar, M. A., \& Tagieva, S. O. 2001, arXiv:astro-ph/0110092.

Allen, M.P. \& Horvath, J.E. 2004, ApJ616, 346

Allen, M.P. \& Horvath, J.E. 2011, arXiv:1009.4498, to appear in the Proceedings of the ASTRONS2010

Conference, Cesme, Turkey, Aug. 2010.

Bibby, J. L., Crowther, P. A., Furness, J. P., \& Clark, J. S. 2008, MNRAS, 386, L23

Burrows, A. \& Nordhaus, J. 2009, in Proceedings of the International Conference in Honor of Dimitri Mihalas for

His Lifetime Scientific Contributions on the Occasion of His 70th Birthday. AIP Conference Proceedings 1171 (NY:AIP), 273

Clark, D. H., \& Stephenson, F. R. 1975, The Observatory, 95, 190

Caswell, J. L., Murray, J. D., Roger, R. S., Cole, D. J., \& Cooke, D. J. 1975, A\&A, 45, 239

Dall'Osso, S., Shore, S.N. \& Stella, L. 2009, MNRAS, 398, 1869

Davies, D. , Figer, D.F. , Kudritzki, R-P., Trombley, C., Kouveliotou, C. \& Wachter, S. 2009, ApJ, 707, 844

Figer, D. F., Najarro, F., Geballe, T. R., Blum, R. D., \& Kudritzki, R. P. 2005, ApJ, 622, L49

Gaensler, B. M., Slane, P. O., Gotthelf, E. V., \& Vasisht, G. 2001, ApJ, 559, 963

Gaensler, B. M., McClure-Griffiths, N. M., Oey, M. S., Haverkorn, M., Dickey, J. M., \& Green, A. J. 2005, ApJ, 620, L95

Halpern, J.P. \& Gotthelf, E.V. 2010a, ApJ, 710, 941

Halpern, J.P. \& Gotthelf, E.V. 2010b, ApJ, 725, 1384 
Hamuy, M. 2003, ApJ, 582, 905

Luz, D. M. G. C., \& Berry, D. L. 1999, MNRAS, 306, 191

Marsden, D., Lingenfelter, R. E., Rothschild, R. E., \& Higdon, J. C. 2001, ApJ, 550, 397

Moiseenko, S. G., Bisnovatyi-Kogan, G. S., Ardeljan, N. V. 2010, in Proceedings of the 4th Gamow International

Conference on Astrophysics and Cosmology After Gamow, AIP Conference Proceedings, 1206 (NY:AIP), 282

Muno, M. P., Clark, J. S., Crowther, P. A., Dougherty, S. M., de Grijs, R., Law, C., McMillan, S. L. W., Morris, M.

R., Negueruela, I., Pooley, D., Portegies Zwart, S., \& Yusef-Zadeh, F. 2006, ApJ, 636, L41

Nomoto, Ken'Ichi, Tanaka, Masaomi, Tominaga, Nozomu \& Maeda, Keiichi, 2010, New Astro. Rev., 54, 191

Ostriker, J. P., \& Gunn, J. E. 1971, ApJ, 164, L95

Rezzolla, L., Lamb, F. K., Markovic, D., \& Shapiro, S. L. 2001, Phys. Rev. D, 64, 104014

Sato, T., Bamba, A., Nakamura, R. \& Ishida,M. 2010, PASJ, 65, L33

Thompson, T. A., Chang, P., \& Quataert, E. 2004, ApJ, 611, 380

Truelove, J. K., \& McKee, C. F. 1999, ApJS, 120, 299

van der Swaluw, E., Achterberg, A., Gallant, Y. A., \& Tóth, G. 2001, A\&A, 380, 309

Vink, J. \& Kuiper, L. 2006, MNRAS, 370, L14

Watts, A. L., \& Andersson, N. 2002, MNRAS, 333, 943

Woods, P. \& Thompson, C. 2006, in Compact stellar X-ray sources. Edited by Walter Lewin \& Michiel van der

Klis (Cambridge Astrophysics Series, No. 39. Cambridge, UK: Cambridge University Press), 547

Woosley, S. 2010, ApJ, 719, L204

Yu, Y-W., Cheng, K.S. \& Cao, X-F., 2010, ApJ, 715, 477 